\title{
¿Qué respiramos? La construcción de saberes y conciencia ambiental entre la universidad y la escuela secundaria, a través de la extensión universitaria
}

\section{What Do We Breathe? Construction of Knowledge and Environmental Awareness Between the University and High School Through University Extension}

Andrea Celeste Isaguirre

Universidad Nacional de San Luis

San Luis, Argentina

ac21isaguirre@gmail.com

https://orcid.org/0000-0003-2414-532X

Mario Franco Moyano

Universidad Nacional de San Luis

San Luis, Argentina

mdmoyano@gmaill.com

D https://orcid.org/0000-0001-8932-202X

Aldo Daguerre

Universidad Nacional de San Luis

San Luis, Argentina

aldobqca@gmail.com

https://orcid.org/0000-0001-8946-8475

Marta Matilde Moglia

Universidad Nacional de San Luis

San Luis, Argentina

mmmoglia@gmail.com

https://orcid.org/0000-0003-4332-6927

Recibido: 11/01/2021 • Aceptado: 30/03/2021

Resumen. El objetivo de este trabajo es abordar una problemática ambiental en relación con la salud humana, para responder a la inquietud presentada por estudiantes y docentes de dos escuelas secundarias de la ciudad de San Luis (Argentina). Esta inquietud se originó a partir del deseo de los actores sociales del 
Revista Universidad en DiÁlogo • Vol. 11, N. ${ }^{\circ} 2$ 2, Julio-Diciembre, 2021 • 141-159

ISSN 2215-2849 • EISSN: 2215-4752

URL: http://www.revistas.una.ac.cr/index.php/dialogo/index CorReo ElECTRÓNICO: universidadendialogo@una.cr

DOI: http://doi.org/10.15359/udre.11-2.7

nivel medio de conocer la calidad biológica del aire que respiran cotidianamente en los ambientes en los que desarrollan sus actividades académicas. A través del trabajo conjunto entre el equipo extensionista de la Facultad de Química, Bioquímica y Farmacia de la Universidad Nacional de San Luis y la comunidad educativa de las escuelas se buscó construir, en un marco dialógico, conocimientos referidos a la educación ambiental.

Durante el desarrollo del presente trabajo se propició la discusión y reflexión acerca del uso de las especies vegetales ornamentales de los espacios verdes de la ciudad y su impacto en la salud pública. La metodología propuesta contempló talleres presenciales para los que se confeccionaron guías teórico-prácticas, adecuadas al nivel académico del grupo de estudiantes, y específicas de las temáticas a abordar. Los conocimientos obtenidos se aplicaron con éxito en actividades experimentales sencillas, relacionadas con las tareas de investigación en aerobiología de la universidad. De esta manera, se logró que el estudiantado fuera partícipe en la generación de conocimiento útil para su beneficio, para el equipo extensionista y para la comunidad de San Luis, en general.

Palabras clave: aire, árboles, contaminación ambiental, diálogo de saberes, extensión universitaria, escuela secundaria.

\begin{abstract}
This work aimed to address an environmental problem related to human health to respond to students and teachers' concerns from two secondary schools in the city of San Luis (Argentina). These concerns originated from secondary school social actors' who desired to know the biological quality of the air they breathe daily in the environments in which they carry out their school activities. Through joint work between the extension team of the Faculty of Chemistry, Biochemistry, and Pharmacy of the National University of San Luis and the school community, it was sought to build knowledge related to environmental education in a dialogic framework. While developing this work, discussion and reflection on the use of ornamental plant species in the city's green spaces and their impact on public health were encouraged. The proposed methodology included face-to-face workshops, for which theoretical-practical guides were prepared, appropriate to the academic level of the students and specific to the topics to approach in this work. The knowledge obtained was successfully applied in simple experimental activities related to research tasks in Aerobiology at the university. Thus, the students could participate in the generation of useful knowledge for themselves, the extension team, and the community of San Luis, in general.
\end{abstract}

Keywords: air, trees, environmental pollution, knowledge dialogues, University Extension, High school. 


\section{Introducción}

Las experiencias cimentadas en la universidad pública argentina se originan como producto y proceso de los dispositivos históricos y culturales particulares en los que la comunidad universitaria fue protagonista, diseñando políticas que orientan la función educativa de la universidad en el presente. "En cada época surgen nuevas incógnitas, que los sujetos se plantean e invitan u obligan a parar, volver a mirar, reflexionar $\mathrm{y}$, si les es posible, proponer" (Wigdorovitz de Camilloni et al., 2013, p. 7).

En el contexto de educar, desde la universidad y para toda la sociedad, cobra fundamental importancia la denominada extensión universitaria. Si bien no hay consenso en relación con la definición de extensión, una de las delimitaciones de su alcance es la señalada por Celman sobre la base de las Resoluciones del Consejo Directivo de la Universidad de la República (2007). En la misma se define a la extensión como

Proceso dialógico y bidireccional, que busca la colaboración entre actores del nivel universitario y actores sociales, en un pie de igualdad, dialogando y combinando sus respectivos saberes al servicio de objetivos socialmente valiosos, dando prioridad a los problemas de los sectores más postergados. (Celman, 2013, p. 9)

No obstante, tal como señala esta misma autora, tradicionalmente se entendió a la extensión como todas las actividades realizadas en el medio social. Así, entre estas se incluyen la difusión o divulgación científica, la transferencia tecnológica y el asistencialismo, actividades que, sin embargo, poseen un enfoque restringido y unidireccional. Por este motivo, el presente trabajo se realizó considerando a la extensión universitaria en el sentido de proceso dialógico bidireccional.

En relación con la extensión universitaria, el Senado y la Cámara de Diputados de la República de la Nación Argentina (1995) promulgó la Ley de Educación Superior (Ley 24.501 de 1995) que manifiesta, en varios de sus artículos, la importancia del vínculo entre la universidad y la sociedad en la cual se encuentra inserta. El fin de esta ley fue atender y responder a las demandas sociales, tanto en los campos educativos como científicos y tecnológicos, en pos de mejorar la calidad de vida y la del medio ambiente, mediante un proceso educativo transformador. 
Revista Universidad en DiÁlogo • Vol. 11, N. ${ }^{\circ} 2$ 2, Julio-Diciembre, 2021 • 141-159

ISSN 2215-2849 • EISSN: 2215-4752

URL: http://www.revistas.una.ac.cr/index.php/dialogo/index Correo eleCtrónico: universidadendialogo@una.cr DOI: http://doi.org/10.15359/udre.11-2.7

Es interesante advertir que la educación universitaria dejó de ser un proyecto individual, para convertirse en un instrumento de la sociedad, para su propia transformación (Wigdorovitz de Camilloni, 2010). Además, puede contribuir a responder a los requerimientos de la sociedad, como institución generadora de personas competentes en la producción de bienes y servicios.

En el marco actual de la vida institucional, en el que una multiplicidad de misiones y funciones se imponen en la universidad, y con mayor intensidad que en el pasado, las funciones de docencia, investigación y extensión deben estar vinculadas de manera estrecha. De gran relevancia es definir cuáles han de ser las voces que deben ser escuchadas y cómo procesa la universidad la multidiversidad de la sociedad, con la que se encuentra en diálogo. Es aquí donde se valorizan las acciones de extensión que se vienen realizando desde hace mucho tiempo en las universidades públicas.

Muchas veces se trata de trabajos de cátedras que están a cargo de un grupo de docentes y que desarrollan acciones en función de algún tipo de demanda que recibieron o, incluso, en virtud de un convenio de servicios. En otras ocasiones, son los mismos equipos universitarios los que se proponen realizar tareas mancomunadas con sectores de la sociedad, en pos de mejorar alguna cuestión ambiental, social, económica, ecológica, etc.

Una de las principales preocupaciones del grupo extensionista en educación es el reconocimiento de los aspectos teórico-pedagógicos que podrían mejorar e incentivar el aprendizaje. En esta tarea se debe ayudar al estudiantado no solo a aprender, sino a prepararse para organizar y supervisar exitosamente su propio aprendizaje. Es decir, para que aprendan a aprender y a potenciar las habilidades y destrezas que cada estudiante posee (Moglia y Daguerre, 2016). En la Biología y otras Ciencias Naturales muchas de las estrategias de enseñanza y aprendizaje, tales como la lectura y el análisis de textos, son comunes entre la mayoría de las disciplinas curriculares.

Otras, como los trabajos de laboratorio, son compartidas solamente con disciplinas como la Química y la Física (Puttick et al., 2015). Particularmente, los trabajos de campo, en los que predomina el enfoque investigador y el contacto con la naturaleza in situ, son característicos de las Ciencias Naturales.

En relación con los aspectos teórico-pedagógicos, la práctica reflexiva se basa en una determinada idea de la profesionalidad, en la que el equipo docente tiene una responsabilidad con respecto a la educación del estudiantado. Esta práctica trasciende el puro instrumentalismo, englobando las responsabilidades 
de educar para la ciudadanía y de imbuir en el alumnado una disposición positiva para el aprendizaje durante toda la vida (Day, 2006).

Por este motivo, las actividades implementadas en esta iniciativa extensionista buscaron desarrollar la reflexión crítica y constructiva, además de promover, a través de estrategias de enseñanza, competencias del estudiantado en el plano experimental (Puttick et al., 2015).

Para desarrollar la extensión, en un sentido correcto, cada extensionista deberá realizar una tarea en común con la comunidad — sin que ello implique hacer asistencialismo- en la que ponga en juego su formación académica y su compromiso ciudadano (Wigdorovitz de Camilloni et al., 2013). Esta posición ubica a la universidad ya no solo como una institución que provee servicios, sino como aquella que construye un marco común de trabajo.

Una sociedad entre la universidad en la que participan no solo docentes, sino también estudiantes de la universidad, en interacción con las comunidades con las que se trabaja. Es por eso que en los proyectos de extensión se debe hablar de socios y no de receptores externos. Así, se propone la generación de una sociedad dentro de la que, en un marco de cooperación, la universidad aprende de las comunidades y estas de la universidad.

\section{Objetivos y propósitos de la propuesta extensionista}

El proyecto de extensión sobre el que se basa el presente trabajo permitió concretar objetivos claros y enriquecedores para los y las partícipes del conocimiento y la responsabilidad ambiental. Como punto principal, se logró amalgamar las tres funciones principales de la universidad: extensión, investigación y docencia.

Se direccionaron las actividades de investigación, desarrolladas por docentes y estudiantes de la universidad que participaron en el mismo, hacia las inquietudes planteadas por actores sociales del nivel secundario, por conocer la calidad del aire que respiran durante sus actividades cotidianas.

El propósito central de la propuesta extensionista pudo adaptar las tareas de investigación, con el fin de que el estudiantado pudiera, a través de experiencias sencillas, resolver sus interrogantes y, a partir de ello, extender sus conocimientos a la comunidad en general. El grupo extensionista aportó toda su experiencia en aerobiología, la rama de la biología que estudia las partículas biológicas presentes en el aire (Galán et al., 2007), y en el conocimiento de los árboles y la forestación urbana responsable. 
Revista Universidad en DiÁlogo • Vol. 11, N. ${ }^{\circ} 2$ 2, Julio-Diciembre, 2021 • 141-159

ISSN 2215-2849 • EISSN: 2215-4752

URL: http://www.revistas.una.ac.cr/index.php/dialogo/index Correo eleCtrónico: universidadendialogo@una.cr DOI: http://doi.org/10.15359/udre.11-2.7

En este sentido, se partió de la información aportada por un proyecto de extensión previo, realizado por el equipo, donde se logró recopilar datos acerca del arbolado urbano, que se volcaron en una aplicación para teléfonos celulares diseñada por estudiantes de una escuela técnica. A través del desarrollo del proyecto, se hicieron extensivos a la comunidad los conocimientos generados en la universidad sobre la contaminación del aire, tanto biológica como por metales pesados, y las enfermedades que ambos contaminantes pueden ocasionar.

Las escuelas integrantes del proyecto poseen diferentes orientaciones que no se incluyen específicamente dentro de las ciencias naturales. Una de ellas posee orientación en ciencias sociales y la otra en educación técnica profesional informática. Los diseños curriculares de la provincia de San Luis, a la fecha de realización del proyecto, no incluían ciclos orientados ni de educación técnica, dado que estaban en construcción; en consecuencia, las escuelas integrantes del proyecto se valieron de los lineamientos nacionales para desarrollar su currículo explícito.

Estas instituciones educativas poseen, asimismo, un vínculo con la universidad a través de docentes en común en ambas instituciones. A su vez, cada escuela contemplaba entre sus objetivos educativos trabajar, a través de disciplinas como la Biología y la Química Orgánica, sobre la educación ambiental, abocada a la concientización y prevención de la contaminación en el ambiente urbano de la ciudad de San Luis.

No obstante, el tipo de contaminación del ambiente contemplada entre los objetivos hacía hincapié, principalmente, en la que ocurre en el agua y en la tierra. En cuanto a la calidad del aire, la inclusión del tema desarrollado en el proyecto de extensión resultó innovadora e integradora, permitiendo analizar el ambiente a nivel de ecosistema.

\section{¿Por qué es importante el ambiente aerobiológico que nos rodea?}

La caracterización aerobiológica del ambiente es importante para conocer las partículas biológicas que lo contaminan y que pueden causar diferentes problemas, como las alergias al polen. En este aspecto, el aire atmosférico no es solo una mezcla de gases, sino que normalmente posee un gran número de partículas contaminantes en suspensión que se depositan finalmente en distintas superficies inanimadas o en los seres vivos.

Estas partículas pueden ser clasificadas como biológicas, orgánicas e inorgánicas (Mandrioli y Comtois, 1998). Entre las partículas biológicas y 
orgánicas se encuentran los granos de polen, las esporas de hongos, los restos vegetales o animales, los microorganismos, etc.

A través de estudios aerobiológicos se puede conocer el contenido de partículas biológicas en el aire, su diversidad y las concentraciones en las que se presentan en las distintas épocas del año (Mandrioli y Comtois, 1998; Galán et al., 2007). Entre las partículas de origen inorgánico existe una amplia diversidad, y unas de las más importantes, debido a los problemas de salud que ocasionan en los seres vivos, son los metales pesados (Hladun et al., 2016).

En la atmósfera exterior es muy frecuente la presencia de granos de polen, especialmente durante la fase de polinación de las plantas. En interior son mucho menos frecuentes estas aeropartículas, mientras que las esporas de hongos son las más abundantes en estos ambientes. Muchas especies de hongos pueden producir, principalmente a través de sus esporas, cuadros de alergias como rinitis, asma, conjuntivitis y, en casos raros, pero más graves, cuadros de inflamación pulmonar, llamados neumonitis (Galán et al., 2007).

Por otra parte, los granos de polen están íntimamente relacionados con el desarrollo de enfermedades alérgicas de tipo respiratorio, incluidas conjuntamente con las causadas por las esporas de hongos, bajo el nombre global de polinosis. Esta patología alérgica puede exacerbarse debido a que las partículas aerobiológicas pueden unirse a sustancias inorgánicas como los metales pesados (Isaguirre et al., 2020).

En relación con la problemática planteada, el presente trabajo resalta el rol extensionista como nexo entre los conocimientos ampliamente desarrollados en el ámbito universitario y las demandas no respondidas sobre aspectos que generan una baja calidad de vida en la sociedad, en relación con los procesos alergénicos y su vinculación con el arbolado urbano.

En este aspecto, las políticas públicas a nivel nacional no tienen especialmente en cuenta la alergenicidad de las plantas utilizadas en la forestación o parquización de lugares públicos, como sería, por ejemplo, el ámbito escolar. En relación con las alergias, la resolución denominada "Normas de Organización y Funcionamiento de la Áreas de Inmunología Clínica y de Alergia" (Ministerio de Salud de la República Argentina, Resolución 158 de 2002) resalta una idea previa sobre la prevención, el diagnóstico y tratamiento de patologías, entre las que se encuentran las alergias respiratorias.

En esta resolución se indican acciones y actividades, como planes de prevención y detección, en las que intervienen diferentes instituciones. San Luis no escapa a 
Revista Universidad en DiÁlogo • Vol. 11, N. ${ }^{\circ} 2$ 2, Julio-Diciembre, 2021 • 141-159

ISSN 2215-2849 • EISSN: 2215-4752

URL: http://www.revistas.una.ac.cr/index.php/dialogo/index Correo eleCtrónico: universidadendialogo@una.cr DOI: http://doi.org/10.15359/udre.11-2.7

la generalidad nacional en cuanto a las escasas políticas públicas en relación con polinosis, aunque, si bien no existen leyes específicas sobre polinosis y arbolado urbano, la Ley IX-0318-2004 promulgada por el Senado y la Cámara de Diputados de la Provincia de San Luis (2004) resalta el Régimen de protección del arbolado público provincial, donde se refieren las normas locales para la protección de los árboles utilizados para la forestación en la ciudad.

\section{Metodología de trabajo}

El proyecto de extensión se desarrolló durante el ciclo lectivo 2019 y se trabajó con los colegios públicos de la ciudad de San Luis (Argentina): Escuela Técnica N. ${ }^{\circ} 7$ "Dr. Manuel Sadosky" y Escuela N. 312 "República de Chile". Las asignaturas implicadas en este proyecto de extensión se relacionan con las Ciencias Naturales: Química Orgánica de $5^{\circ}$ año y Biología de $4^{\circ}$ año.

El grupo extensionista estuvo integrado por docentes con formación en Ciencias Biológicas y Bioquímica y por estudiantes avanzados de las carreras del Profesorado en Biología y Licenciatura en Bioquímica. Los/as integrantes universitarios/as del proyecto compartieron con el estudiantado los conocimientos obtenidos a partir de la investigación sobre aerobiología y contaminación con metales pesados.

Los y las estudiantes del Profesorado Universitario en Biología, dado sus conocimientos referidos tanto a cuestiones específicas de la disciplina como a aquellas vinculadas con la enseñanza de las ciencias, pudieron participar más activamente en una experiencia, a modo de práctica profesional.

De esta manera, a través de la inclusión de prácticas extensionistas en el estudiantado, se logró un acercamiento a la curricularización de la propuesta de extensión (Hernández, 2020). Esta propuesta logró que se enfrentaran a grupos de estudiantes reales, en escuelas públicas de la ciudad, resultando en una experiencia de gran importancia para su actividad laboral futura.

La metodología de trabajo contempló talleres presenciales, durante los cuales se proporcionaron los conocimientos teóricos necesarios para comprender la temática de la contaminación del aire por partículas biológicas y metales pesados. Además, se rescataron los conocimientos del estudiantado, los cuales enriquecieron la experiencia didáctica. Esta primera etapa de capacitación permitió proyectar la realización de actividades de laboratorio. Un paso fundamental en la misma fue la confección de guías teórico-prácticas que fueron entregadas al alumnado, y que estuvieron apoyadas por recursos audiovisuales. 
Estas actividades previas permitieron que el grupo de alumnos y alumnas obtuviera los conocimientos necesarios para la identificación del polen y el reconocimiento de los árboles que ocasionan polinosis y están presentes en su entorno. Asimismo, el grupo de estudiantes del nivel secundario aportó sus experiencias familiares y personales acerca del conocimiento sobre los árboles que actualmente están presentes en la ciudad de San Luis y sobre los múltiples malestares alérgicos que pueden ocasionar sus pólenes, que, en algunos casos y según relatos del alumnado, impedían el desarrollo normal de su vida cotidiana.

Las actividades didácticas de tipo experimental permitieron que el estudiantado lograra identificar y reconocer las partículas aerobiológicas presentes en la atmósfera de los lugares donde transcurren sus actividades habituales. Para el desarrollo de estas jornadas se utilizó la metodología propuesta por Moglia y Daguerre (2016), que consistió en colocar dispositivos de muestreo en lugares a elección, como el patio del colegio, las aulas, la biblioteca, etc.

Los dispositivos fueron conformados por portaobjetos a los cuales se les adhirió una cinta Melinex impregnada con silicona transparente, donde quedaron depositadas las partículas aerobiológicas. Luego, a la cinta se le colocó un cubreobjetos impregnado con glicerogelatina-fucsina, que tiñe específicamente granos de polen y restos vegetales.

En la segunda jornada, el grupo de estudiantes de secundaria realizó una visita al Laboratorio de Biología de la Universidad Nacional de San Luis, donde realizó una lectura de las muestras recolectadas, utilizando un microscopio óptico. En esta oportunidad, el grupo de jóvenes conoció cómo funcionan los captadores aerobiológicos, además de poder indagar acerca de la temática y observar sus propias muestras. También, se realizó un encuentro para que el estudiantado conociera la metodología y el instrumental que se utiliza para la determinación de metales pesados.

Cada encuentro, si bien tuvo momentos meramente prácticos en los que predominó el uso de instrumental de laboratorio, también ofreció debates en torno a la problemática ambiental de la ciudad de San Luis. Estos debates propiciaron un intercambio de opiniones en referencia a las posibles soluciones que se pueden dar para mitigar, al menos en parte, las consecuencias del mal uso de árboles alergénicos en los espacios urbanos.

Los planteamientos de ideas por parte del estudiantado, como referentes de las zonas en las que viven, aportaron a la comunidad universitaria un panorama más amplio acerca del tipo de vegetación que existe en diversas zonas de la ciudad y de los problemas que causan a la comunidad. 
Revista Universidad en DiÁlogo • Vol. 11, N. ${ }^{\circ} 2$ 2, Julio-Diciembre, 2021 • 141-159

ISSN 2215-2849 • EISSN: 2215-4752

URL: http://www.revistas.una.ac.cr/index.php/dialogo/index CORREO ELECTRÓNICO: universidadendialogo@una.cr DOI: http://doi.org/10.15359/udre.11-2.7

Cabe aclarar que las jornadas desarrolladas en ambas instituciones fueron organizadas y autorizadas en forma conjunta entre docentes extensionistas de la Universidad Nacional de San Luis y el equipo docente-directivo de las escuelas secundarias.

\section{Interrogantes abordadas con los y las estudiantes de secundaria}

Como inicio de la ejecución de este proyecto, docentes y estudiantes del nivel universitario confeccionaron una guía teórico-práctica que fue entregada a cada estudiante, donde se encontraba la fundamentación teórica de la importancia del estudio de la calidad del aire. Asimismo, se presentó la guía práctica para la toma de muestras y un instructivo sobre el uso adecuado del microscopio, instrumento de gran importancia para la realización de la parte práctica de este proyecto. Además, se informaron las medidas de bioseguridad pertinentes para trabajar en el laboratorio. A continuación, se mencionan algunos ejemplos de los debates llevados a cabo entre el equipo extensionista y el estudiantado del nivel secundario:

\section{¿Qué contiene el aire que respiramos?}

Si bien muchos y muchas estudiantes tenían conocimiento sobre la composición química del aire, su inquietud radicaba en el hecho de que los libros de textos, si bien aportan esa información, no profundizan acerca de los contaminantes que puede presentar, entre los que se encuentran partículas biológicas, orgánicas y no biológicas.

Frente a esta reflexión, el grupo de jóvenes planteó que consideraba que muchas patologías se transmiten y propagan a través del aire, por lo que sería oportuno conocer qué otros constituyentes están presentes en el mismo, más allá de la simple mezcla de gases que indican los libros. Fue así que, a raíz de estos cuestionamientos, se decidió comenzar a incorporar algunos conocimientos acerca del contenido aerobiológico de la atmósfera: granos de polen, esporas de hongos, fragmentos de vegetales, de insectos, fibras como microplásticos, etc.

Además, se mencionó el plomo como producto de la combustión de los motores de los autos de la ciudad y otros elementos como el manganeso, el cobalto, el bario y el cadmio como contaminantes presentes en el aire que se respira y que, muchas veces, causan problemas de salud. 


\section{¿Qué son los granos de polen?}

Es aquí donde el equipo extensionista desarrolló, en un primer momento, el concepto simple de polen como el polvillo amarillo que presentan las flores y sobre su participación en la reproducción sexual de las plantas. En este punto, se aprovechó para aclarar las ideas previas erróneas que tenía el alumnado en relación con el polen como la gameta masculina que fecunda a la gameta femenina en las plantas y, sobre estos conceptos, construir el nuevo conocimiento.

Para asombro de muchas de las personas participantes, utilizando imágenes se les explicó que el polen no es la gameta masculina, sino que es un gametofito, portador de las gametas masculinas (células espermáticas), que son las que van a fecundar a la célula femenina (oósfera). A partir del reconocimiento de la estructura del polen como el polvillo amarillo, que todos y todas habían observado alguna vez, se amplió el concepto señalando que: si se realiza una vista microscópica del mismo, se podrá observar que cada grano se trata de una estructura diminuta, que tiene forma y tamaño característicos, dependiendo de la planta de la que provenga.

Se trató de enfocar el concepto de que plantas emparentadas, por ejemplo, del mismo género o incluso familia, tendrán, en general, granos de polen similares. También se informó acerca de la existencia de plantas que poseen flores con granos de polen muy parecidos a los de otras especies no emparentadas y que esto constituye un problema para su identificación. No obstante, si se conoce el momento en que se produce la polinación de cada una de ellas, se pueden diferenciar, en general, los granos de polen presentes en el aire, para un determinado tiempo y espacio.

El desafío de conocer todo ese universo que escapa al ojo desnudo dio lugar a la interrogante que presentó el estudiantado... ¿por qué es importante saber qué tipos de granos de polen existen en el aire? La respuesta a esta pregunta estuvo directamente vinculada con la aparición de procesos alérgicos, en función a los granos de polen que puedan existir en el ambiente. De esta premisa surgió otra incógnita clave que fue desarrollada:

\section{¿Es importante tener en cuenta el tipo de plantas a utilizar para ornamentar las ciudades?}

En relación con este interrogante, una parte fundamental de las tareas del equipo extensionista fue indagar los conocimientos del estudiantado sobre las plantas que existen en los lugares donde realizan sus actividades cotidianas y profundizarlos. Se destacó el gran beneficio, en materia ambiental, de la 
Revista Universidad en DiÁlogo • Vol. 11, N. ${ }^{\circ} 2$ 2, Julio-Diciembre, 2021 • 141-159

ISSN 2215-2849 • EISSN: 2215-4752

URL: http://www.revistas.una.ac.cr/index.php/dialogo/index CORREO ELECTRÓNICO: universidadendialogo@una.cr DOI: http://doi.org/10.15359/udre.11-2.7

presencia de espacios verdes en las ciudades, pero se enfatizó en que el uso de especies ornamentales debía llevarse a cabo con precaución y responsabilidad.

Usualmente, las plantas son utilizadas para la ornamentación de espacios verdes, tanto públicos (plazas, bulevares, parques, etc.) como privados (jardines y patios internos de viviendas). Pero, al momento de elegir qué especies utilizar, sería importante tener en cuenta la posibilidad de que algunas de ellas pueden ser nocivas para la salud. Se resaltó que hay que ser consciente de que algunas especies, por más estéticas que sean, pueden producir alergias en la población o incluso ser tóxicas y que, en consecuencia, es de gran importancia planificar la forestación de los espacios urbanos.

Asimismo, se remarcó que hay que tener en cuenta que todas las plantas que poseen flores masculinas (con órganos sexuales masculinos: las anteras) son productoras de polen. Entre los tipos o las formas biológicas más comunes de las plantas se incluyen a los árboles (plantas leñosas con un tallo no ramificado), las plantas arbustivas (con tallos leñosos ramificados desde la base) y las hierbas (plantas herbáceas, como las gramíneas o las margaritas, que no poseen tallos leñosos - no poseen madera-).

Por otro lado, se mostró que entre las plantas ornamentales existen algunas nativas u originarias de San Luis, y otras que son exóticas. Es decir, que no son originarias de esta región, sino que fueron introducidas por el ser humano. En los espacios verdes urbanos muchas de las plantas que se encuentran son exóticas y se cultivan en los mismos debido, por ejemplo, a la sombra que proporcionan o a la belleza que aportan al lugar.

Se remarcó asimismo que, generalmente, los árboles exóticos cultivados, como moras, fresnos y cipreses, producen más alergia que aquellos nativos, como los algarrobos o los quebrachos blancos, debido a que producen pólenes muy pequeños y en gran cantidad. Esto reviste importancia al momento de la elección de ejemplares que van a ser utilizados para forestar la ciudad. Así, la decisión de colocar unas u otras especies influirá en el desarrollo de alergias al polen u otros problemas de salud en las personas que integran la comunidad.

\section{Poniendo en práctica lo aprendido}

Luego de la exposición, donde se llevó a cabo la presentación de los objetivos y la fundamentación teórica del proyecto, así como también la de sus integrantes, el grupo de estudiantes comenzó a elaborar los dispositivos para la toma de muestras de aire, con la ayuda del equipo extensionista. 
Posteriormente, el grupo de estudiantes colocó los dispositivos armados en cajas de plástico, en los distintos ambientes que ellos frecuentan dentro de la escuela: polideportivo, patio, entrada del colegio y aula (allí los dejaron por siete días).

Transcurrido el tiempo de muestreo, el estudiantado retiró las cajas de plástico de los lugares seleccionados. En la jornada posterior, realizada en el Laboratorio de Biología de la Universidad Nacional de San Luis, el grupo de alumnos y alumnas llevó las muestras recolectadas y las colorearon con fucsina. Esta coloración les permitió la observación de los granos de polen, utilizando un microscopio óptico, además de conocer cuáles eran los tipos polínicos que estaban presentes en los diferentes ambientes que frecuentan.

En el Laboratorio de la Universidad Nacional de San Luis, el estudiantado también realizó la extracción, preparación de muestras y observación de polen fresco de diversas especies arbóreas cercanas a la universidad, lo que les permitió conocer otros tipos de granos de polen frecuentes en muestras aerobiológicas.

A partir de sus resultados, y de los nuevos conocimientos adquiridos sobre aerobiología, el alumnado pudo concluir cuáles eran, dentro de su institución educativa, los lugares que debían evitar en caso de poseer alergias conocidas a determinados pólenes. Se proyectó la difusión a la comunidad de los resultados obtenidos por el estudiantado durante esta experiencia extensionista, y se planearon encuentros similares en otras instituciones educativas y de salud, uno de los cuales actualmente se encuentra aprobado para su ejecución.

\section{Análisis de la experiencia}

Las actividades planteadas en este proyecto de extensión se centraron en la recreación dinámica de actividades prácticas experimentales de aerobiología, fundamentadas en el diseñar y el hacer conjunto entre extensionistas, docentes y estudiantes de la comunidad educativa secundaria. En relación con los trabajos prácticos de laboratorio, tales como los que clásicamente se conocen, no permiten que el grupo de estudiantes aborden la naturaleza de la ciencia y ofrecen muy pocas oportunidades para la exploración auténtica de fenómenos.

Así, si bien existe una variada gama de posibilidades en la implementación de trabajos prácticos de laboratorio (Fernández, 2013), en general, se desarrollan a través del seguimiento de guías, a las que se ha comparado con "recetas de cocina", en las que el estudiantado sigue fielmente las indicaciones escritas, desaprovechando así el potencial que este recurso posee (Merino y Herrero, 2007). 
Revista Universidad en DiÁlogo • Vol. 11, N. ${ }^{\circ} 2$ 2, Julio-Diciembre, 2021 • 141-159

ISSN 2215-2849 • EISSN: 2215-4752

URL: http://www.revistas.una.ac.cr/index.php/dialogo/index Correo eleCtrónico: universidadendialogo@una.cr DOI: http://doi.org/10.15359/udre.11-2.7

En consecuencia, queda poca o nula opción para el estudiantado de experimentar y reflexionar por sí mismo (Carp et al., 2012). Por estas razones, se sugiere que, entre otras cosas, el aprendizaje debe ser reforzado a través de un incremento de la colaboración profesional entre profesores/as e investigadores/as y del rol activo del equipo extensionista.

Las técnicas que se utilizaron para "emprender" la extensión, así como aquellas que se emplearon para desarrollar las actividades incluyeron desde la formulación hasta la puesta en práctica del proyecto como una tarea entretejida de la docencia-investigación-extensión. No se puede obviar que la extensión ha sido pensada históricamente como "transferencia" del saber que se gesta en la universidad hacia aquellos sectores que no tienen acceso al mismo y que, como personas ciudadanas, tienen derecho a ello.

Esta premisa quedó significada al poder romper fronteras de la desigualdad, especialmente en una de las instituciones educativas secundarias con la que se trabajó, ubicada en un entorno socioeconómico postergado. Fue reconfortante para docentes y estudiantes "poner en práctica" la teoría académica o los conocimientos provenientes del ámbito experimental (Schneider, 2018).

Prevalece la idea de extensión universitaria gestada antes y durante la Reforma Universitaria de 1918, que estableció la democratización del saber y del conocimiento científico, siendo esta una manera de inclusión social. Este concepto pragmatizado como una de las funciones de toda universidad contribuye significativamente a resignificar y mejorar los procesos de enseñanza y aprendizaje, elevando la calidad educativa de las instituciones universitarias (Menéndez, 2017).

Desde este proyecto se puso en acción a la extensión como una de las funciones sociales de la universidad. Así, se pusieron los saberes académicos en diálogo con otros saberes, los cuales, a través de ese encuentro, se enriquecieron mutuamente. Este es un ejemplo de lo que se ha denominado extensión crítica, según lo expresado por Tommasino y Cano (2016), con el sentido humanista y pedagógico de extensión que propone Paulo Freire (1987).

Este proyecto tomó como punto de partida las ideas previas del grupo de estudiantes sobre los problemas de salud generados por el "reverdecer" de las plantas en época primaveral o por los fenómenos meteorológicos de vientos o sequías, como desencadenantes de muchos trastornos respiratorios. El desafío implicó postular y defender la posición que sugiere Ranciére (2007), quien considera que algunos saberes, si bien son provenientes de la educación formal, no son más importantes o necesarios que otros no formales. 
En la extensión, justamente, diversos saberes se encuentran y entran en conflicto para potenciarse (Schneider, 2018). Así, a partir de los conocimientos y las ideas previas del grupo de estudiantes sobre el aire, las plantas, sus pólenes y los factores meteorológicos, a los que se sumaron los conocimientos adquiridos a partir de las experiencias realizadas, se fue construyendo un nuevo conocimiento sobre las características aerobiológicas del ambiente de San Luis y sus posibles efectos sobre la salud.

Asimismo, a través de este proyecto se logró cumplir con los objetivos propuestos, tomando en cuenta los siguientes aspectos:

- Se realizaron actividades claras y motivadoras para el estudiantado, uso de guías teórico-prácticas, muestreo ambiental y visita a la universidad.

- Hubo una participación conjunta de docentes y estudiantes universitarios/as con docentes y estudiantes de secundaria, de forma coordinada en la búsqueda de conocimiento.

- Se impulsó el aprendizaje experiencial, el cual es una estrategia de enseñanza con enfoque holístico que está destinada a relacionar el aprendizaje académico con la vida real. Con ese fin, se propuso al estudiantado realizar actividades en las que, a partir de esa conexión con la práctica, ponga a prueba las habilidades y los conocimientos teóricos que posee, evalúe sus consecuencias, enriquezca esos conocimientos y habilidades e identifique nuevos problemas. Así, cuando el aprendizaje es experiencial, quien aprende lo hace en las condiciones sociales de la utilización auténtica de los conocimientos. Aprende en un marco en que se apunta al logro simultáneo de fines comunitarios y fines educativos (Wigdorovitz de Camilloni, 2016).

De esta manera, en la experiencia educativa basada en una correcta transición nivel medio-nivel universitario, se pudo observar que resulta de vital importancia despertar la atención y el razonamiento del estudiantado por medio de la aplicación práctica del conocimiento científico a casos reales, a través del mundo de la experimentación, logrando un aprendizaje pleno (Perkins, 2010).

Se potenció el fortalecimiento de la extensión, concebida como un "proceso dialógico y bidireccional", que buscó la colaboración entre actores universitarios $\mathrm{y}$ actores del nivel secundario en un pie de igualdad, dialogando y combinando sus respectivos saberes al servicio de objetivos socialmente valiosos. 
Revista Universidad en DiÁlogo • Vol. 11, N. ${ }^{\circ} 2$ 2, Julio-Diciembre, 2021 • 141-159

ISSN 2215-2849 • EISSN: 2215-4752

URL: http://www.revistas.una.ac.cr/index.php/dialogo/index Correo eleCtrónico: universidadendialogo@una.cr

DOI: http://doi.org/10.15359/udre.11-2.7

El grupo de estudiantes del nivel medio reaccionó con gran entusiasmo, aportando respuestas dinámicas y de gran utilidad para su formación intelectual, a partir de los conocimientos básicos aportados por los y las docentes de la universidad, implicándose en la construcción de su propio conocimiento y produciendo a su vez conocimiento útil para el equipo extensionista y para la comunidad local.

Asimismo, el estudiantado logró potenciar sus destrezas en el manejo de instrumental especializado, como el microscopio, que no es utilizado habitualmente en las escuelas, por no contar con el mismo o por poseer una cantidad insuficiente. Las experiencias sencillas desarrolladas por el alumnado les permitieron indagar sobre aspectos desconocidos, como la identificación de partículas aerobiológicas.

Además, pudieron enriquecerse a través del trabajo en equipo, buscando saberes útiles para la vida cotidiana y académica. Las actividades de tipo experiencial permitieron que el grupo de estudiantes desarrollara capacidades críticas y creativas de forma activa, incentivando el conocimiento y el aprendizaje.

En este sentido, el conocimiento crítico, que se pretendió impulsar durante el proyecto, se construye y se elabora a través de procesos intelectuales y motrices que implican realizar asociaciones, relaciones y abstracciones y formular conclusiones, análisis o síntesis, de forma activa y consciente (Jara, s. f.).

\section{Conclusiones}

El trabajo se enfocó en estimular el compromiso del estudiantado en la solución de los problemas de la comunidad, consolidando sus conocimientos a través del intercambio y contacto con el mundo donde se inserta cotidianamente. Su importancia radicó en promover el aprendizaje ambiental a través del análisis de una situación problemática real como la polinosis.

El proceso implicó la transposición didáctica de conocimientos académicos universitarios y la construcción de otros nuevos, partiendo de las ideas y experiencias previas que el grupo de estudiantes tenían acerca de la temática abordada. Asimismo, contribuyó a la construcción de una postura crítica, factible de instrumentalizarse socialmente, frente a un problema ambiental vigente. Es así como, a través de la participación como actores sociales responsables, en sus entornos habituales, el estudiantado se convirtió en agente diversificador de los saberes construidos en una actividad educativa. 
El grupo de jóvenes de las escuelas no solo brindó el conocimiento acerca de lo que ocurre en materia ambiental en sus entornos, sino que también lo hizo con su visión crítica acerca del tratamiento de espacios públicos. Esto, sin duda, favorece la labor en investigación aerobiológica de otros ambientes de la ciudad, así como también la referida a la extensión universitaria, apostando a sumar mayor cantidad de escuelas para que realicen esta actividad.

Esto último se justifica en la importancia del estudiantado como vehículo del saber, en pos de la transformación social respecto de la educación ambiental, propiciando la mejora de los espacios públicos. Así, transmitirán, entre las personas con las que se vinculan, aquellos conocimientos referentes a la importancia de un parquizado urbano responsable.

Por otra parte, la incorporación y activa participación en todas las instancias del estudiantado universitario en el proyecto permitieron asimismo amalgamar docencia, investigación y extensión. De esta manera, las actividades de extensión realizadas contribuyeron, en alguna medida, a recuperar la función social de la universidad.

\section{Referencias}

Carp, D., García, D. y Chiacchiarini, P. (2012). Trabajos prácticos de laboratorio sin receta de cocina en cursos masivos. ACI, 3(1), 167-173.

Celman, C. (2013). La extensión como espacio político y pedagógico. En G. Menéndez (ed.), Integración, docencia y extensión. Otra forma de enseñar y aprender (pp. 5-10). Universidad Nacional del Litoral, Argentina.

Consejo Directivo Central de la Universidad de la República. (Setiembre, 2007). Resoluciones del Consejo Directivo de la Universidad de la República. Uruguay.

Day, C. (2006). Pasión por enseñar. La identidad personal y profesional del docente y sus valores. Madrid: Narcea.

Fernández, N. E. (2013). Los trabajos prácticos de Laboratorio en la enseñanza de la Biología. Revista de Educación en Biología, 16(2), 15-30.

Freire, P. (1987). La educación para la práctica de la libertad. Buenos Aires: Siglo XXI Editores. 
Revista Universidad en DiÁlogo • Vol. 11, N. ${ }^{\circ} 2$ 2, Julio-Diciembre, 2021 • 141-159

ISSN 2215-2849 • EISSN: 2215-4752

URL: http://www.revistas.una.ac.cr/index.php/dialogo/index CorReo ElECTRÓNICO: universidadendialogo@una.cr DOI: http://doi.org/10.15359/udre.11-2.7

Galán, C., Cariñanos, P., Alcázar, P. y Domínguez, E. (2007). Manual de calidad y gestión de la Red Española de Aerobiología. Editorial Argos Impresores.

Hernández, M. (2020). La vinculación universidades-comunidades como generadora de conocimiento. Revista Universidad en Diálogo, 10(2), 125-142. https://doi.org/10.15359/udre.10-2.6

Hladun, K. R., Di, N., Liu, T. y Trumble, J. T. (2016). Metal contaminant accumulation in thehive: consequences for whole colony health and brood production in the honey bee (Apis mellifera L.). Environ. Toxicol. Chem., 35, 322-329.

Isaguirre, A. C, Moyano, M. F., Gil, R. A. y Moglia, M. M. (2020). A novel and simple method for elements determination in aerobiological samples by Inductively Coupled Plasma Mass Spectrometry (ICPMS) analysis. Water, Air and Soil Pollution, 231(2), 70-79. 10.1007/ $\underline{\mathrm{s} 11270-020-4416-2 .}$

Jara, O. (S. f.). La concepción metodológica dialéctica, los métodos y las técnicas participativas en la educación popular. San José, Costa Rica: Centro de Estudios y Publicaciones Alforja.

Mandrioli, P. y Comtois, V. (1998). Methods in Aerobiology. Editorial Pitagora.

Menéndez, G. (2017). Resignificación de la extensión a 100 años de la Reforma Universitaria de 1918. Revista + E versión en línea, 7(7), 24-37.

Merino, J. M. y Herrero, F. (2007). Resolución de problemas experimentales de Química: una alternativa a las prácticas tradicionales. Revista Electrónica de Enseñanza de las Ciencias, 6(3), 630-648.

Ministerio de Salud (2 de abril, 2002). Resolución 158. Normas de Organización y Funcionamiento de la Áreas de Inmunología Clínica y de Alergia. República Argentina.

Moglia, M. y Daguerre, A. (2016). ¿Inspirar seres vivos? Una propuesta didáctica integradora para introducir a los estudiantes en la investigación en biología a través de la aerobiología. KIMÜN Revista Interdisciplinaria de Formación Docente, 2, 202- 215.

Perkins, D. (2010). El aprendizaje pleno, principios de la enseñanza para transformar la educación. Argentina: Editorial Paidós. 
Puttick, G., Drayton, B. y Cohen, E. (2015). A Study of the Literature on LabBased Instruction in Biology. The American Biology Teacher, 7(1), 12-18.

Ranciére, J. (2007). El maestro ignorante. Cinco lecciones sobre la emancipación intelectual. Libros del Zorzal

Schneider, G. (2018). Extensión y etnografía: una travesía por la integralidad y la comprensión de prácticas de comunicación comunitaria. $+E$ : Revista de Extensión Universitaria, 8(9), 157-166.

Tommasino, H. y Cano, A. (2016). Modelos de extensión universitaria en las universidades latinoamericanas en el siglo XXI: tendencias y controversias. Universidades, 67, 7-24.

Wigdorovitz de Camilloni, A. (2010). La formación de profesionales en la universidad. Gestión Universitaria. Revista Electrónica de la Universidad Nacional de La Matanza, 2(2). http://www.gestuniv.com.ar.

Wigdorovitz de Camilloni, A., Rafaghelli, M., Kessler, M., Menéndez, G., Boffelli, M., Sordo, S., Pellegrino, E. y Malano, D. (2013). Integración, docencia y extensión: otra forma de enseñar y de aprender. Universidad Nacional del Litoral, Argentina.

Wigdorovitz de Camilloni, A. (2016). Tendencias y formato en el currículo universitario. Itinerarios Educativos, 9, 11-26. 